\title{
CCD image sensor induced error in PIV applications
}

\author{
M Legrand ${ }^{1}$, J Nogueira ${ }^{1}$, A A Vargas ${ }^{1}$, R Ventas ${ }^{1}$ and M C Rodr' Iguez-Hidalgo $^{2}$
}

\section{Introduction}

During the past century, scientific imaging has proven its utility and capability in numerous fields. It covers the whole range from the largest observable objects in astronomy down to the smallest ones in microscopy. It also assists critical domains of modern society, such as medical imaging and environmental protection. Although photographic film technologies were an important scientific breakthrough and allowed for many studies, the recent developments of digital imaging enhance the capacities to a great extent. This is especially noticeable when dealing with image processing and storage. Among other applications, these improvements are a relevant benefit in most scientific research domains.

In particular, this evolution has allowed remarkable enhancement of particle image velocimetry (PIV) as an image based, non-intrusive, and global velocimetry technique widely used in the field of fluid mechanics and thermal engineering. At its beginning, it was common to use pulsed illumination to record the particle image positions twice in a photographic film at different times, separated by a small time interval $\Delta t$. Although relevant studies and results were obtained (Kompenhans and Reichmuth 1986, Lourenço et al 1986, Grant and Smith 1988, Cho 1989, among others), the 


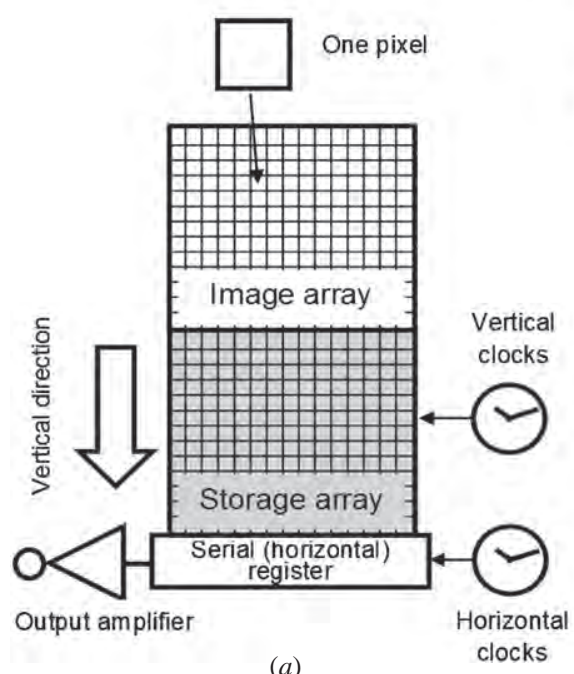

(a)

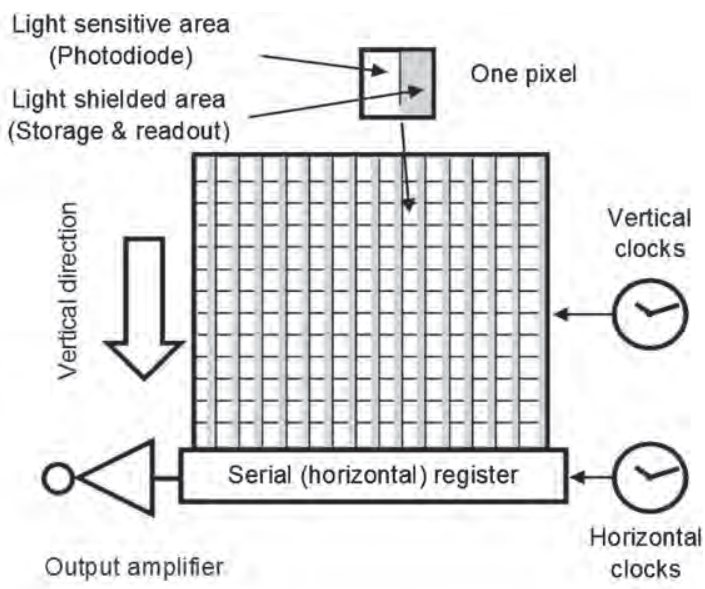

(b)

Figure 1. General CCD architectures: frame-transfer $(a)$ and interline-transfer $(b)$. The light sensitive area can also be envisaged as a capacitor in CCD technology; here the nomenclature 'photodiode' (Kodak 2011, among others) is used instead.

interrogation techniques, such as Young fringe analysis, were very delicate and time consuming (Grant 1997). In addition, the quality of the experimental images could not be checked until developing the film, hours after the acquisition. Another drawback of this analog technique was that the photographic film was exposed twice, requiring specific devices to avoid directional ambiguity. Since the early 1990s (Willert and Gharib 1991, Wernet 1991), the use of charge-coupled device (CCD) image sensors allowed for digital recording of particle position, greatly easing the image processing. It is usual to operate the CCD cameras in a double-frame mode, i.e. acquiring one different frame by exposure, thus solving the directional ambiguity problem.

The CCD image sensors have experimented great improvements since the early 1980s. In the framework of particle image velocimetry (PIV), the interline-transfer technology allowed us to achieve very small interframe times, down to $\sim 100 \mathrm{~ns}$, enabling the measurement of fluid flows with a fast movement relative to the field of view.

Among the many lines of study in the use of CCD cameras in PIV, Nogueira et al (2009) and Legrand et al (2011) recently reported that the double-frame CCD camera readout process present some relevant bias in the location of particle images, thus biasing the velocity measurement. Both works deal with the bias impact on the measurement error, and propose a way to evaluate its magnitude using a simple multiple $\Delta t$ strategy, available for any usual PIV setup, in the image acquisition stage. Together with preliminary references from Raffel et al (1998), the aforementioned studies attribute a displacement error to the CCD readout procedure, but generally there is a lack of information on the phenomenology of this bias error. With the exception of Nogueira et al (2009), who reported some evidence of CCD illumination influence, no explicit dependence on internal or external variables is usually given. The present study is dedicated to unveiling such dependences in order to further characterize the CCD readout error, including the possible effect of temperature
(Waczynski et al 2001) and ambient humidity, as suggested by Nogueira et al (2009). This work also allows prediction of when strategies such as the 'multiple $\Delta t$ ' one, may be appropriated to correct this kind of error in the measurements, thus providing an additional tool to the researchers interested in designing a PIV acquisition protocol that optimizes the degree of accuracy of a PIV measurement.

\section{Frame-transfer and interline-transfer CCD image sensors}

\subsection{Sensor architecture: frame-transfer versus interline-transfer}

Since its beginning, digital imaging, and CCD sensors in particular, has experienced great evolution and enhancement. Given the large variety of application fields, several different designs have emerged, depending on the particular requirements. As commented in the introduction, the implementation of PIV requires acquiring two single exposure frames in a short, or very short, interval of time $\Delta t$. For stateof-the-art CCDs, the minimum time to transfer (read out) all the pixels of a typical megapixel CCD sensor is about $100 \mathrm{~ms}$, far above the $\Delta t$ needed in many applications (typically of the order of few microseconds or even less than that). This requirement has been met with the development of cameras with two CCD arrays implanted in the acquisition chip.

The so called frame-transfer technology consists in two identical rectangular CCD arrays, where one of them is protected from the incident light by a shield (generally a thin aluminum coating). With this arrangement (see figure 1 $(a)$ ) only one CCD array is able to collect light: the image array. When a first exposure is finished, all the photon-generated charges in the elements (pixels) of this CCD array are simultaneously transferred to the second, light shielded, CCD: the storage array. Once the whole image has been transferred to the storage zone, taking $\sim 10$ to $100 \mu$ s depending on the design, a second exposure may start in the, now empty, image 


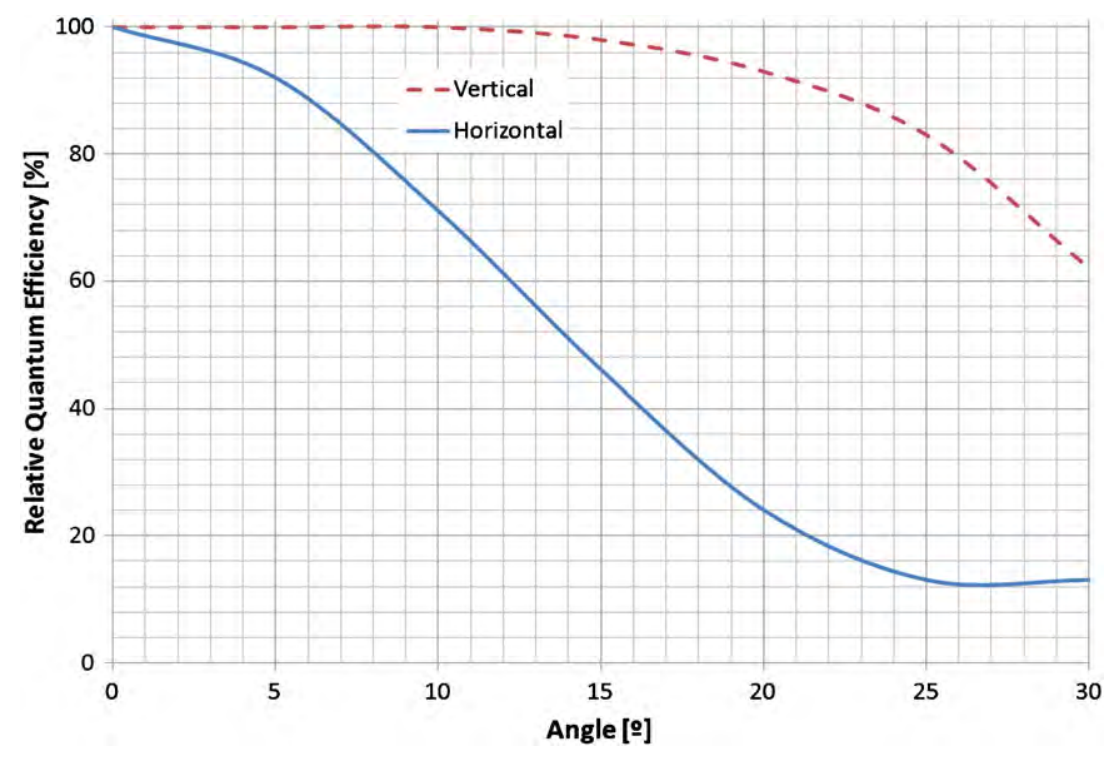

Figure 2. Relative quantum efficiency versus azimuthal angle (horizontal corresponds to the direction across interlines) of the Kodak KAI4011 4 megapixel interline CCD sensor. The difference is mainly due to the micro-lenses, photodiodes and CCD storage element layout.

array. During the storage array readout $(\sim 50 \mathrm{~ms})$, the second exposure takes place. As the frame transfer operation is much faster than the readout, a shorter $\Delta t$ between exposures is possible.

Interline-transfer CCDs (or interline CCDs for short) were developed to avoid some of the shortcomings of the frametransfer devices (figure $1(b)$ ) by locating the photo-detecting and storage elements closer than in the previous case. This is done by inserting photosensitive element lines in between lines of storage non-sensitive (light shielded) elements. These storage lines are also called vertical or parallel registers, as they are used also in the readout procedure to drive the information towards the serial (horizontal) register. Since each photodiode (light sensitive area in figure $1(b)$ ) is adjacent to one CCD storage unit, in this configuration the interline transfer is much faster than in frame transfer technology, reaching interframe times down to $\sim 100 \mathrm{~ns}$. This simple operation achieves the transfer of the entire exposed image into the adjoining storage sites. The fast transfer, in conjunction with the now current higher pixel density formats, has extended the field of applications, including its common application to velocity measurement techniques such as PIV. The major disadvantages of the interline CCD layout are a lower sensitivity per surface unit and a higher complexity, leading to higher unit costs. Among other reasons, the lower sensitivity occurs because at each pixel about half the area is needed for the interline storage array (fill factor $\mathrm{FF} \sim 0.5$ ). This last issue requires the use of micro-lenses mounted on top of the photodiodes forming vertical strip prisms. This configuration is useful to overcome the low fill factor, FF, in the serial direction, but it produces a strong dependence on light incidence angle, giving quite different sensitivities in vertical (parallel) and horizontal (serial) directions. Figure 2 presents the performances of the Kodak KAI4011 interline CCD provided with micro-lenses (Kodak 2011).

In the rest of the paper, the interline CCD architecture is the one further analyzed and studied. Its short interframe time for a double-frame acquisition has made it the most frequent choice for PIV applications.

\subsection{Interline pixel architecture and readout procedure}

In addition to the error model described in section 2.3 and later, this subsection contains some details and references to the interline pixel architecture. This can be useful for readers interested in studying possible improvements of the mentioned model. Figure 3 offers a simplified layout of a true 'two phase buried channel' interline transfer CCD with a 'pinned photodiode' pixel architecture. In this nomenclature, 'pinned photodiode' means that the n-doped layer of the sensor is underneath a thin heavily p-doped layer that pins its potential (see the photodiode cross sections in figure 3). This allows for a larger charge capacity and better blue response (details in Burkey et al (1984)). 'Buried channel' indicates that the voltage gates (blue and orange in figure 3 ) of the vertical (parallel) register act over an n-doped buried channel instead of directly over the substrate (again the photodiode cross sections in figure 3 show this detail). 'Two phase' means that this register completes a charge shift after two timing clock steps following a procedure that is detailed further below in this subsection (figure 4). This device (Janesick and Putnam 2003, among others) is commonly used in high performance cameras oriented to PIV image acquisition. The layout of figure 3 shows the photosensitive area (photodiode) where an incident photon produces an electron-hole pair in the $\mathrm{p}^{+}-\mathrm{n}$ junction by the photoelectric effect. The generated electrons migrate away from the junction, towards the potential well in the n-doped zone of the photodiode. Here the electrons are accumulated, or in other terms time-integrated, during the exposure time. The incident light is thus converted into electrical charges in a proportional way. If the illumination is too high or the exposure too long, the charge integration reaches the full well capacity, leading to saturation, which should be avoided. The overflow drain (OD, Kodak 2008) device, included to avoid 
Top view

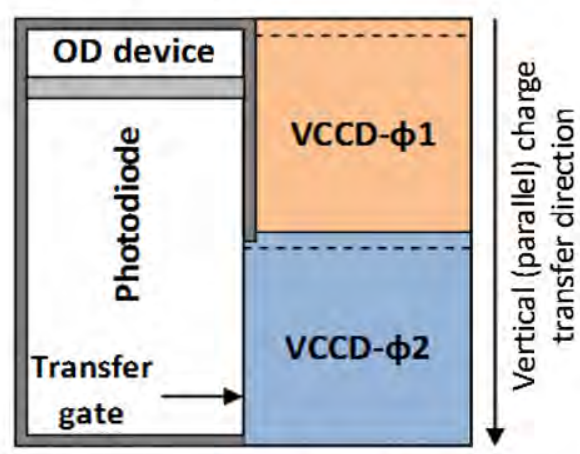

Cross section through photodiode and CCD register V1

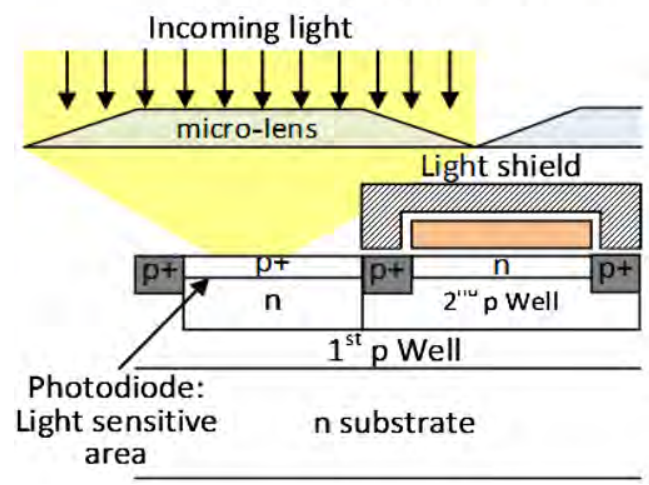

\section{Cross section down through}

CCD register

Vertical (parallel) charge transfer

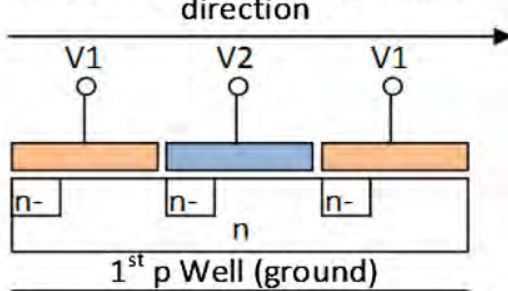

n substrate

\section{Cross section through photodiode} and CCD register $\mathbf{V} \mathbf{2}$ at transfer gate

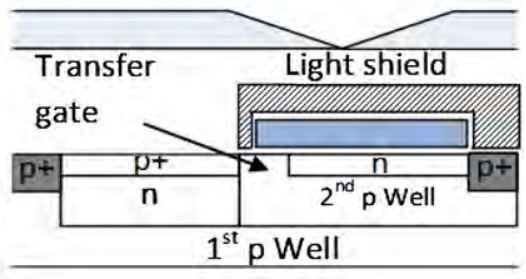

n substrate

Figure 3. True two phase buried channel interline CCD pixel architecture showing OD and micro-lens arrangement (metal and oxide layers are not shown for simplicity).

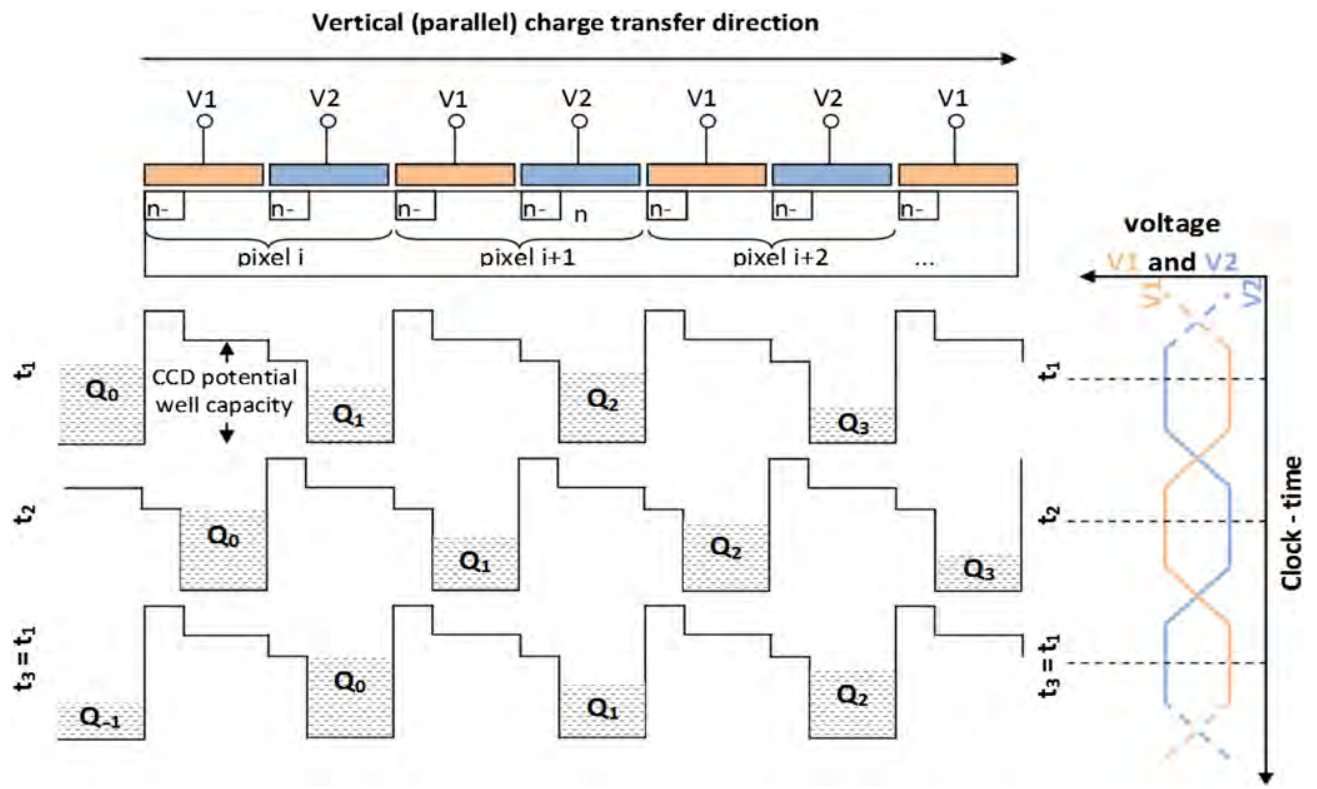

Figure 4. Vertical CCD shift register, V1 and V2 potentials and clock timing.

spillage of saturated charges to neighbor CCD array elements, is also shown. A description of the key points in the readout procedure is given below.

The photodiode is insulated from the next pixel by a heavily doped $\mathrm{p}^{+}$potential barrier to avoid the contamination of neighbor pixels. Figure 3 depicts this barrier in dark gray for easy identification. Adjacent to the photodiode, the CCD register, or storage area, is separated into two distinct areas, VCCD- $\Phi 1$ and VCCD- $\Phi 2$ (where V indicates vertical layout of these elements in the CCD), connected to different voltage gates: V1 and V2, respectively. Narrow regions of weakly negatively doped $\mathrm{n}^{-}$material lie between the parallel transfer 


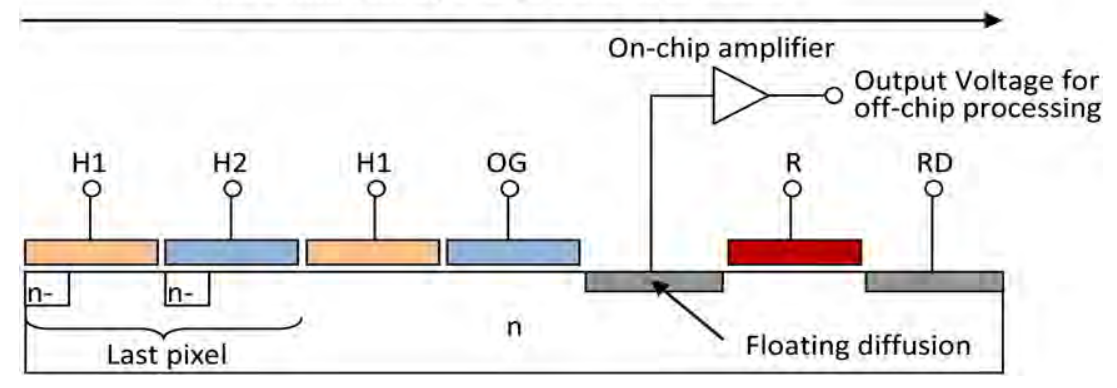

Figure 5. Horizontal shift register and sense node.

electrode strips. These regions in the buried channel are known as channel stops and are in charge of repelling the electrons generated in the $\mathrm{CCD}$, thus preventing electrons from wandering to another adjacent pixel on the CCD, as figure 4 details.

The photodiode and the VCCD- $\Phi 2$ are connected together through a transfer gate, which opens at the end of the first exposure in order to allow the extremely fast interframe charge transfer into the CCD register for storage. Cross sections through the photodiode and the CCD storage registers show the built-in light shield on top of the CCD register region. A simplified micro-lens arrangement is also shown. The lens cross section remains constant along the vertical direction, accounting for the directional quantum efficiency differences exposed in figure 2. According to their vertical design, VCCD$\Phi 1$ and VCCD- $\Phi 2$ areas constitute the CCD vertical shift registers and allow the vertical readout of the image sensor.

Summarizing the working procedure of these CCDs, during the first exposure, the light gathered by each single pixel is integrated as electrical charges in the potential well of the photodiode. In a second step, the accumulated charges carriers at each pixel are simultaneously (all at once) transferred to the adjacent $C C D$ register through a transfer gate at VCCD- $\Phi 2$ (see figure 3 ). Then the readout of the whole CCD registers starts. This instant corresponds to internal clock time $t_{1}$, as shown in figure 4 . At clock time $t_{2}$, the vertical (parallel) shift clock triggers the transfer of all the charge from VCCD- $\Phi 2$ to the next VCCD- $\Phi 1$, applying the voltages shown in figure 4 for V1 and V2. Next, at clock time $t_{3}, \mathrm{~V} 1$ and V2 are set again to the voltages indicated at time $t_{1}$. This figure also unveils how the 'channel stops' prevent charges from being trapped or left behind.

At this point, all horizontal pixel lines have been shifted one row downward, so that the lower line enters the serial shift register (figure $1(b)$ ), responsible for horizontal readout. The horizontal clock shifts all the pixels of that line, one at a time, in an analogous way to the vertical shift procedure, for the whole line. This is done by sequentially applying voltages $\mathrm{H} 1$ and $\mathrm{H} 2$ to the $\mathrm{CCD}$ register gates (figure 5). Horizontal clocking is not a 'true two phase' one, but rather a 'pseudotwo phase' clocking (Kodak 2008, Theuwissen 1995) in order to enable the charge transfer in both directions. This is very useful for large chips, as separating the horizontal readout into two halves almost reduces the line timing by half, enhancing CCD readout time performance.
Figure 5 illustrates the charge transfer and readout in the serial (horizontal) CCD register. As the charge packets are transported through this register, the last charge packet of the line is dumped on the output sense node, where the electrons are converted to a voltage that is easier to work with for the rest of the electronics, in short, 'off-chip'.

Conventional techniques usually employ a floating diffusion region for this output sense node. Floating diffusion consists of a gate-free node (i.e. no potential is imposed on it), thus it is separated from the last $\mathrm{H} 1$ gate by the output gate $(\mathrm{OG})$ in order to avoid parasitic potentials. The $\mathrm{OG}$ is a constant, low voltage gate generating a potential barrier meant to keep the charge packet waiting while the floating node is being reset. At that time, the reset gate $(\mathrm{R})$ is positively biased to clamp the floating diffusion region to the reset drain potential (RD). Then the charge packet is transferred to the floating diffusion through the output gate. The change in potential between RD and the charge packet is sensed as a voltage through a high gain capacitance connected to the floating region. It is worth to notice that in the sensing process (charge to voltage conversion) the charge packet remains less than half the horizontal clock period, thus being the smallest transfer time in the whole chip. In addition, the distance between the last $\mathrm{H} 1$ gate and the floating diffusion is larger than the gate to gate distance (figure 5 is not to scale).

Once the whole line has been read out through the floating diffusion node, via the on-chip amplifier, a new parallel shift occurs, thus repeating the whole process again, until the entire CCD array is read out.

\subsection{Readout smearing}

When transferring electrical charges through the serial or parallel registers, it is essential that the whole of the charge packet moves to the next electrode; otherwise the image integrity would be lost. Unfortunately, the charge transfer is not perfect, and some charges may leak out of the potential wells by diffusion (among other complex phenomena out of the scope of this study), generating some smearing of the image acquired by the CCD. For PIV applications, the images usually consist of a dark background with bright dots corresponding to particle scattering. Smearing of these images along a privileged orientation generates undesirable error in the location of the particles. Departure from perfect charge transfer efficiency (CTE), due to small traps in the silicon structure, may generate 
the mentioned smearing. The traps extract a small amount of charge and release it at a later time, thus transferring the charge into the following charge packet. Thus, the CTE is dependent on clock frequency. It is generally $99.9999 \%$ (charge transfer inefficiency is hence CTI $=1-$ CTE $\sim 10^{-6}$ ) for vertical shift registers, but somewhat less for horizontal shifts $\left(\mathrm{CTI} \sim 10^{-5}\right.$ ) because of the necessary higher operating rate (a whole line has to be read out until the next vertical shift can occur, thus limiting the whole process speed). Finally, CTI at the floating diffusion node is suspected to be the largest one, since charge packets are driven here for the smallest amount of time in the whole readout circuit.

Smearing that can be explained by CTI has been found in PIV images in the past; its tendency to deform particle images generates biases on the particle image location as large as 0.2 pixels (Nogueira et al 2009). Fortunately, for PIV applications, both the first and second exposures are smeared in the same direction, reducing the error induced on velocity measurements. However, charge transfer efficiency is not identical for the first and for the second image capture readout, leading to a residual differential bias in the digitally registered particle displacement. This bias error has already been observed and its magnitude evaluated for particular cases by Nogueira et al (2009) and Legrand et al (2010, 2011).

\subsection{Other sources of error considered}

There are other error sources within the readout process that have been studied in this work. The study indicates that they do not have a predominant role in contemporary PIV errors. Nevertheless, they are briefly commented on below. The first one is related to the fact that some interline architectures could suffer from image lag as a consequence of a non-perfect charge transfer from the photodiode to the CCD storage area, i.e., some charges keep trapped in the photodiode. This could yield ghost images in the second exposure. Fortunately, this last issue is almost inappreciable in most recent CCDs, offering image lags of the order of $10 e^{-}$with respect to full well capacity (typically $40000 e^{-}$).

The CCD device can saturate when overexposed. The charges $\left(e^{-}\right)$are generated in proportion to the collected light. If the potential well of the photodiode does not have the capacity to hold the charge created by the photoelectric effect, it will 'bloom' or spill into the adjacent active areas, corrupting the image information. Almost all recent interline transfer CCDs incorporate anti-blooming devices. As an example, an overflow drain (OD for short) is depicted in figure 3. It is separated from the photodiode potential well by a potential barrier. The barrier between photodiode and OD is designed to a lower level than the barriers between pixels, so that collected charge exceeding this level spills vertically through the silicon and is swept away through the $\mathrm{n}$ substrate. Although these devices allow for anti-blooming, they do not prevent saturation occurring, which would obviously bias the PIV correlation (Lecuona et al 2004) if present.

Even below the saturation exposure (e.g. 60\%), some light optical leakage to the adjacent vertical CCD register can occur in addition to the readout errors. This leakage can be produced by different mechanisms: direct incident light on the CCD register, refraction by concave lenses redirecting light on the CCD register instead of the photodiode, and the waveguide effect of multiple reflections and refractions through the lens and silicon layers (Teranishi and Ishihara 1987). However, these effects are largely eliminated in conventional interline devices (typically below $0.01 \%$ as commented by Janesick and Putnam (2003)).

\section{Displacement bias error in PIV applications}

Although different mechanisms are involved in image smearing artifact, (i.e. CTI, image lag, blooming and light leakage), the major effect is attributed in this paper to the CTI, especially at the floating diffusion node. This hypothesis relays on the observation that the amount of smearing does not depend on the location of the particle image within the CCD. As a consequence, the CTI at intermediate transfers does not seem to be relevant compared with the one at this node. Upon this assumption, the authors elaborate a simple model to predict the amount of smear produced during image frame readout.

Other scenarios may be present in special conditions. For space mission cameras where CCDs are more exposed to radiation, as reported by Whitmore et al (1999) and Waczynski et al (2001), energetic particles such as those from cosmic rays can generate additional electron traps in the silicon structure. This increases the CTI at intermediate transfers. Also, other sources of smearing such as CCD image binning, that has been reported to strongly bias particle centroids position (see, e.g., Kholmatov et al 2010), are left out of the scope of this work.

\subsection{Readout error modeling}

Charge transfer efficiency CTE is generally defined as the amount of transferred charges $I$ in a single register shift with respect to the accumulated charges $I_{0}$ before the shift. CTI is then the complementary part, simply defined as CTI = 1 - CTE. Waczynski et al (2001) reported that CTI was sensitive to the integrated charge packet $I_{0}$. Based on the results from that work, CTI has been modeled as shown:

$$
\begin{gathered}
\mathrm{CTI}=1-I / I_{0}=L \cdot I_{0}^{-a} \\
\mathrm{CTI}=\left(I_{\mathrm{ref}} / I_{0}\right)^{a}
\end{gathered}
$$

where $L$ is a charge loss coefficient and $a$ corresponds to a positive value. To emphasize the physical meaning of this equation, this paper uses the normalization given in equation $(1 b)$ rather than the nomenclature of equation $(1 a)$. Clearly, $I_{\text {ref }}=L^{(1 / a)}$.

Equation (1) shows that more illumination (larger value of $I_{0}$ ) implies a smaller CTI. The results of Waczynski et al (2001) show that $a \sim 2 / 3$ for the tested interline-transfer CCDs. This reference also tested deteriorated CCDs irradiated by high energy proton beams (simulating space mission camera irradiation) at several irradiation levels. The parameter $a$ remained almost constant $(a \sim 0.65 \pm 15 \%)$, whereas $L$ was growing for more deteriorated CCDs. Equation (1) also 
indicates that CTI would be different for the first and second exposure images if the collected light $I_{0}$ were different.

As CTI is expected to be larger at the floating diffusion gate, the charge transfer process is simulated at that point. It is assumed that the trapped charges at floating diffusion are left behind to form part of the next pixel readout. $\mathrm{CTI}^{2}$ terms are neglected, leading to equation (2) for the charge $I_{i}$ sensed for pixel $i$. It depends on the accumulated charge $I_{0, i}$ at this pixel location, and on the released charges coming from the previously read out pixel $(i-1)$ :

$$
I_{i}=I_{0, i} \cdot\left(1-\mathrm{CTI}_{i}\right)+I_{0, i-1} \cdot \mathrm{CTI}_{i-1}
$$

In usual PIV applications, the CCD camera operates in a double frame mode, registering seeding particle image positions at two different times, separated by an interval $\Delta t$. With this information, the displacement of particle clusters is estimated by local cross correlations (Raffel et al 1998) between the two frames. If $I_{0}$ were the same for the particle image at both first and second frame exposures, the images would be smeared by the same amount and the effect of this readout process deficiency on the measured displacement would be minimized (except for a small subpixel interpolation error due to the correlation peak shape, but this can be assimilated to a peak locking error (Westerweel 2000, Nogueira et al 2001, 2009, 2011, Legrand et al 2011)).

Unfortunately, in PIV applications, light sources are difficult to operate with exactly the same amount of light for each shot. In order to achieve two pulses in the very brief $\Delta t$ time interval, two laser heads are typically required. Besides the difficulty of tuning both lasers to emit the same amount of energy, the Gaussian profiles of the two laser beams are not identical and may generate some local illumination differences across the region under study. In addition, displacement of particles within the Gaussian profile between the two exposures would generate illumination differences even in a perfectly laser sheet tuned PIV experiment (Nobach and Bodenschatz 2009, Nobach 2011). Thus the relative illumination difference $\Delta I_{0} / I_{0}$ seems to be a key parameter to take into account when studying the displacement bias errors.

To evaluate the effect of this source of error on a PIV measurement, 1D Gaussian particle images of $d_{p}=$ 2.2 ( $^{-2}$ diameter) are used as a first approximation. A CCD fill factor FF $=1$ is considered for simplicity. The first exposure particle image has a maximum intensity $I_{\max , 1}=I_{\max }+\Delta I_{\max } / 2$, while the second one has $I_{\max , 2}=$ $I_{\max }-\Delta I_{\max } / 2$. The illumination difference is thus defined as $\Delta I_{\max }=I_{\max , 1}-I_{\max , 2}$, whereas $I_{\max }$ is the average maximum particle intensity $I_{\max }=\left(I_{\max , 1}+I_{\max , 2}\right) / 2$. As the first particle image location relative to the entire pixel plays an important role in PIV errors (Legrand et al 2011), particle images have been located from -0.5 to +0.5 pixels with respect to the pixel center. In addition, real PIV images generally present a non-zero background, mostly accounting for background illumination/laser reflections and the CCD's dark current effect. To reproduce this issue, a constant background level $I_{\text {noise }}=8$ counts has been added to all the pixels of the generated particle images. Then, the generated 1D particle images are artificially smeared following equation (2), and this is performed for both exposures. Finally, the smeared particle images are crosscorrelated (via FFT and no image deformation, aiming at simplicity), using a three-point Gaussian sub-pixel peak fitting algorithm to find the correlation maximum, and from this the estimated displacement is calculated. The process is repeated for 51 different first particle image locations in the CCD (ranging from -0.5 to +0.5 pixel), and then the average is computed. Then the difference between this value and the real displacement is obtained, giving the error estimation. These calculations have been performed for different particle displacements ranging from 0 to 1 pixel (homogeneous distribution). The results include the error coming from this modeling of the readout error coupled with the unavoidable peak-locking phenomenon emerging from the chosen peak fitting function and the lack of resolution of the CCD sensor to describe small particles (Nogueira et al 2009, 2011, Legrand et al 2011). In order to identify the contribution corresponding to the CCD readout error, the mentioned calculations were compared with those obtained from the same procedure but excluding the simulation of the readout error, described in equation (2). The results indicate that the bias error from the CCD readout for a particle displacement of 0 pixels is a good reference for any other displacement. The magnitude of this error, namely the displacement bias error, $B$, is shown in figure 6 for the mentioned displacement of 0 pixels. For the rest of the possibilities tested (i.e. displacements ranging from 0 to 1 pixel), the curves in figure 6(a) may displace by \pm 0.01 pixels up or down, but the slope practically does not change.

Figure $6(a)$ depicts the results of this error evaluation for different image illumination intensities $I_{\max } / I_{\text {ref }}$ and for different relative illumination differences $\Delta I_{\max } / I_{\max }$ between the two exposures. For a constant $I_{\max } / I_{\text {ref }}$, the displacement bias error $B$ is almost proportional to the relative illumination difference $\Delta I_{\max } / I_{\max }$.

In figure $6(a)$, the average slope of the curves depends on the maximum normalized collected light $I_{\max } / I_{\text {ref }}$, being proportional to $\left(I_{\mathrm{ref}}^{1 / 2} / I_{\max }^{3 / 2}\right)$. A third parameter to take into account is the particle image diameter $d_{\mathrm{p}}$. The previous evaluation was expanded to particle image diameters ranging from 1.25 to 3.25 pixels and different charge loss coefficients ( $a$ and $I_{\text {ref }}$ ) as well as to a range of noise levels $I_{\text {noise }}$. This allows the error behavior to be revealed in respect to the different variables. These results from this model can be combined to obtain the dimensionless correlation proposed in equation (3) and the plot of figure $6(b)$ :

$$
\begin{aligned}
& \frac{B}{\text { pix }} \sim\left(1.5-0.17 \cdot \frac{d_{p}}{\text { pix }}\right) \cdot \frac{\Psi}{\Phi} \\
& \text { where } \\
& \Psi=\left(\left(1-\frac{\Delta I_{\max }}{2 I_{\max }}\right)^{-1.13 a}-\left(1+\frac{\Delta I_{\max }}{2 I_{\max }}\right)^{-1.13 a}\right) \cdot\left(\frac{I_{\text {ref }}}{I_{\max }}\right)^{1.13 a}
\end{aligned}
$$

and

$$
\begin{aligned}
\Phi= & 0.87+1.4\left(\frac{I_{\text {ref }}}{I_{\text {noise }}}\right)^{2} \\
& +\left(0.37-2.5\left(\frac{I_{\text {ref }}}{I_{\text {noise }}}\right)^{2}\right)\left(\frac{I_{\text {ref }}}{a \cdot I_{\text {max }}}\right)^{2} .
\end{aligned}
$$




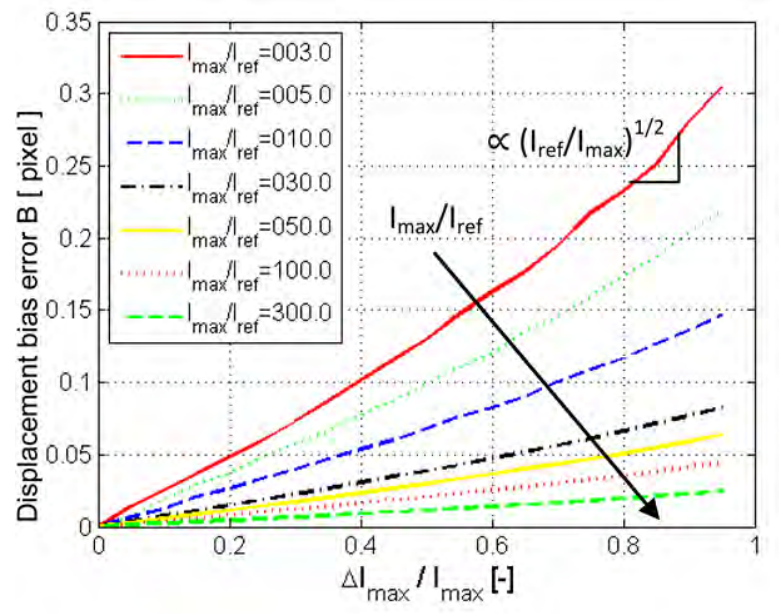

(a)

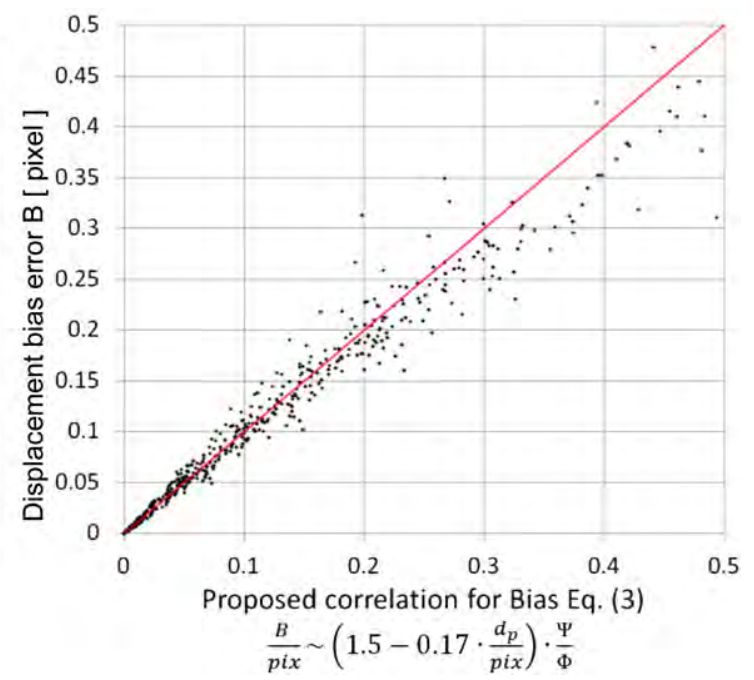

(b)

Figure 6. Readout displacement bias error as a function of relative difference illumination between two exposures, for Gaussian profile particles $(\mathrm{FF}=1)$ and for different maximum intensities $I_{\max }\left(I_{\text {ref }}=18.7(L=5) ; a=0.55.\right)(a)$ Case for real displacement $=0$ and particle diameter 2.2 pixels. (b) Modeled bias error displacement versus proposed correlation in equation (3).

Correlation range: $0.35<a<0.75 ;\left|\frac{\Delta I_{\max }}{I_{\max }}\right|<1 ; \frac{I_{\mathrm{ref}}}{I_{\max }}<0.5$; $\frac{I_{\text {noise }}}{I_{\text {ref }}}<0.5 ; 1.25<d_{\mathrm{p}}<3.25$. In this expression, 'pix' indicates the pixel side length.

In real images, $I_{\text {noise }}, I_{\max } \Delta I_{\max }$ and $d_{\mathrm{p}}$ have to be evaluated, while $a$ and $I_{\text {ref }}$ are camera parameters that have to be fitted to match the correlation proposed in equation (3). In this work, $I_{\text {noise }}$ is evaluated as the rms of the background of the $I \times J$ image: $I_{\text {noise }}=\sqrt{\frac{\sum_{i=1}^{l} \sum_{j=1}^{J} I_{\text {noise }, i, j}^{2}}{N^{2}}}$. On the other hand, the particle image diameter $d_{\mathrm{p}}$ has been estimated as the $\mathrm{e}^{-1}$ diameter of the images autocorrelation peak (which corresponds to the $\mathrm{e}^{-2}$ particle diameter if both are Gaussian shapes). Finally, $I_{\max }$ is estimated on a series of $k$ images as $I_{\max }=\max _{k}\left(I_{i, j, k}\right)-I_{\text {noise }}$ in order to match the model parameters described in this section.

The results from figure 6 and equation (3) allow for a displacement bias error prediction in PIV measurements, but the constants $I_{\text {ref }}$ and $a$ have to be fitted for each particular PIV camera in order to obtain reliable predictions. This is the objective of the next subsection, together with the validation of the proposed correlation.

\subsection{Displacement bias error in real PIV CCD cameras}

In order to validate the previous error modeling and expression (3), an experimental test procedure that uses real images has been designed. The camera used for this validation is the 'MegaPlus' model ES 4.0/E of 4 megapixels, 12-bit dynamic range, incorporating the Kodak KAI-4000M CCD sensor, provided with micro-lenses (Kodak 1999). The general architecture and clock timing correspond to the description in section 2. In addition, the CCD is divided into two halves of 2048 pixels $\times 1024$ pixels, with corresponding parallel shifts: the top region performs upward vertical shifts of 1024 rows, and the bottom half does this downwards. Serial shifts at both ends of the vertical shift registers are also divided into two

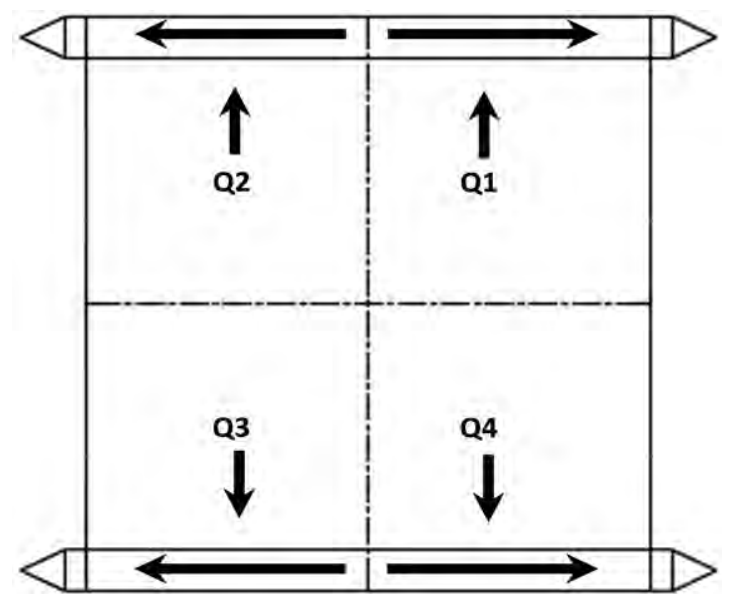

Figure 7. Kodak KAI-4000M image sensor simplified layout.

parts in order to diminish the readout time and thus enhance the frame rate. This is performed using the pseudo-two phase timing, already commented on in section 2. A sketch of the general KAI-4000M layout is shown in figure 7 . Such a readout arrangement results in four on-chip amplifiers and the resulting four video outputs, one for each quadrant $\mathrm{Q}$ (numbered in figure 7).

In addition to the relative illumination difference between two exposures, the experimental procedure includes the evaluation of possible temperature and ambient humidity effects. These could be relevant variables as reported by Waczynski et al (2001) and suggested by Nogueira et al (2009), and sometimes harsh experimental environments determine the working conditions of the cameras in industrial wind tunnels or open facilities.

In general, the CCD temperature may be different from the ambient one. CCD chips are cooled by Peltier cells in order to enhance their sensitivity (quantum efficiency) and reduce thermal noise (dark current). However, most of these Peltier 


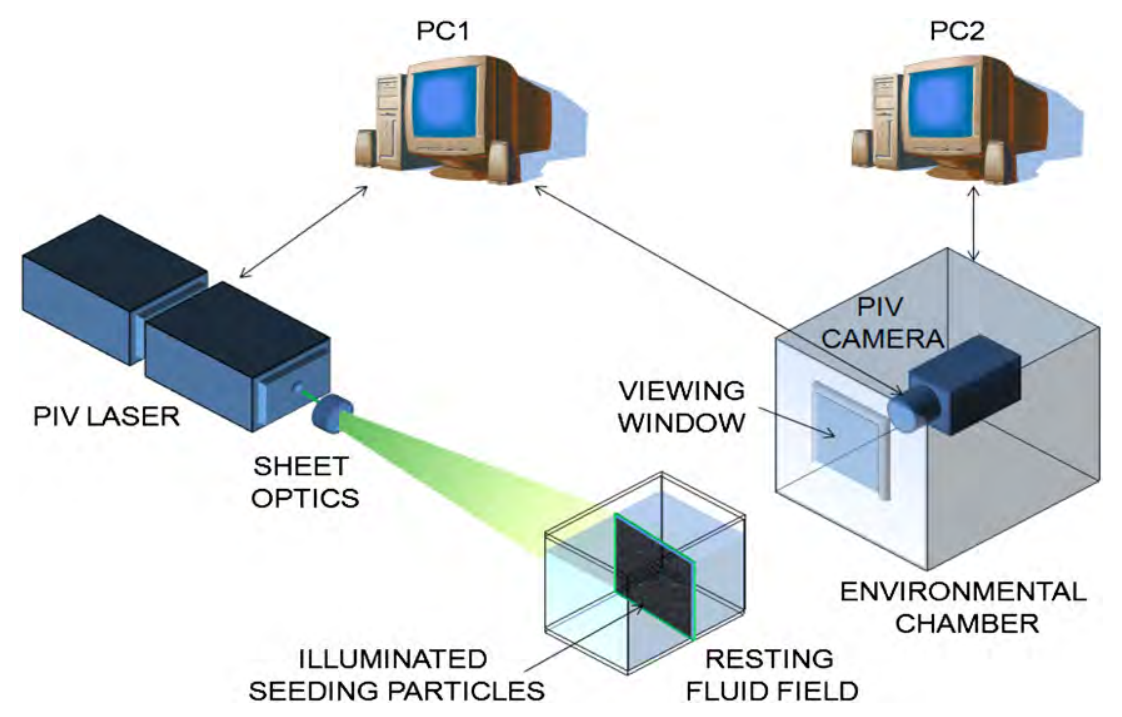

Figure 8. Experimental setup.

cells are operating against ambient temperature, without thermal control, achieving basically an almost constant temperature drop $\Delta T$ respect to the ambient. This is the case of the tested camera.

The experimental setup is described in figure 8. An environmental chamber, controlled by a PC, maintains a constant temperature (within $\pm 1 \mathrm{~K}$ ) and relative humidity (within $\pm 5 \%$ ). The PIV camera is located inside this chamber, and it is synchronized with the PIV laser operation thanks to another PC. The environmental chamber viewing window enables the camera to acquire images of the illuminated seeding particles $\left(d_{\mathrm{p}}=10 \mu \mathrm{m}\right)$ suspended in quiescent water. With the experimental setup magnification and diffraction limited spot, the diameter of the particle image has been estimated to be $d_{\mathrm{pi}} \sim 16 \mu \mathrm{m}$, corresponding to $\sim 2.2$ pixels $\left(\frac{2.44 f \#(1+M) \lambda[\mu \mathrm{m}]}{\operatorname{pixel~size}[\mu \mathrm{m}]}\right)$.

The time interval between the two laser pulses $(\Delta t)$ is set to ensure that the particle displacements to measure are smaller than $\sim 10^{-3}$ pixel (i.e., particles practically do not move). In this condition, illumination differences between the two laser pulses generate different particle image intensities for each exposure. Following the rationale of the model in section 3.1, this induces different smearing along the readout direction for each exposure. This last difference is measured by the PIV cross correlation, providing a measurement of the spurious displacement bias error $B$. In the different test setups, the local relative illumination differences $\Delta I_{\max } / I_{\max }$ range from -20 up to $60 \%$ (see figure 11). The magnitude of the bias error, $B$, can reach 0.15 pixels, as figure 10 shows.

The PIV measurements have been repeated for two MegaPlus CCD cameras, for different temperatures $(\{5,10,15$, $\left.20,25\}{ }^{\circ} \mathrm{C}\right)$, different relative humidities $(\{40,60,80\} \%)$, and different laser energies $(\{50,70,90\} \%$ of maximum nominal shot energy $(380 \mathrm{~mJ}$ at $532 \mathrm{~nm})$ ). For each measurement condition, a series of 200 image couples was acquired in order to achieve reliable average statistics. Once the images were acquired, the maximum intensities for each image couple $\left(I_{\max , 1}\right.$ and $I_{\max , 2}$ ) were computed. The displacement field (bias error $B$ ) was computed through PIV cross correlation, using
$64 \times 64$ pixels $^{2}$ interrogation windows, with $50 \%$ overlap. The ensemble average vector field displacement was finally calculated for each PIV grid location $(64 \times 64)$ for each 200 image couple series. Because of the overlapping of the interrogation windows, the border vectors were not taken into account, resulting in $31 \times 31$ effective vector maps at each quadrant.

In these tests, temperature and relative humidity effects are negligible when compared with the effect of $\Delta I_{\max } / I_{\max }$ and $I_{\max } / I_{\text {ref. }}$ Variations due to temperature and humidity are smaller than $\sim 0.015$ pixels, so their effect has been neglected in the rest of the paper. Nevertheless, it is worthwhile to mention that at $5{ }^{\circ} \mathrm{C}$ the camera has shown many operation problems, in particular when operating at high relative humidity. These data points are not shown in figure 9, as PIV correlation only yielded spurious vector calculations due to the high level of image deterioration.

Figure 9 shows the results obtained for the first camera at each PIV grid location of the first CCD quadrant (Q1 in figure 7) for 200 image couples at each of the 36 measurement setups (four temperature, three humidity and three laser intensity measurement conditions). As data present some dispersion, a moving average filter (64 points) has been included in the plot to highlight the linear data tendency. In particular, figure $9(a)$ shows the result of the model discussed in section 3.1 for horizontal (serial) shift, with $I_{\text {ref }}=18.7$ (i.e. a charge loss factor $L=5$ ), and an exponent $a=0.55$. An excellent agreement between modeling and experimental results can be appreciated, validating the model described in section 3.1. Figure $9(b)$ shows the displacement bias error, but this time in the vertical (parallel) direction. As commented in the previous section, it is much smaller than the serial one (by a factor of $\sim 30$ ).

Figure 10 depicts the results of the horizontal (serial) displacement bias error for both cameras. Here, each quadrant data have been averaged in order to present a single point for each measurement condition. The results are coherent with the one presented in the previous figure, but they unveil the behavior of each one of the four quadrants for each 


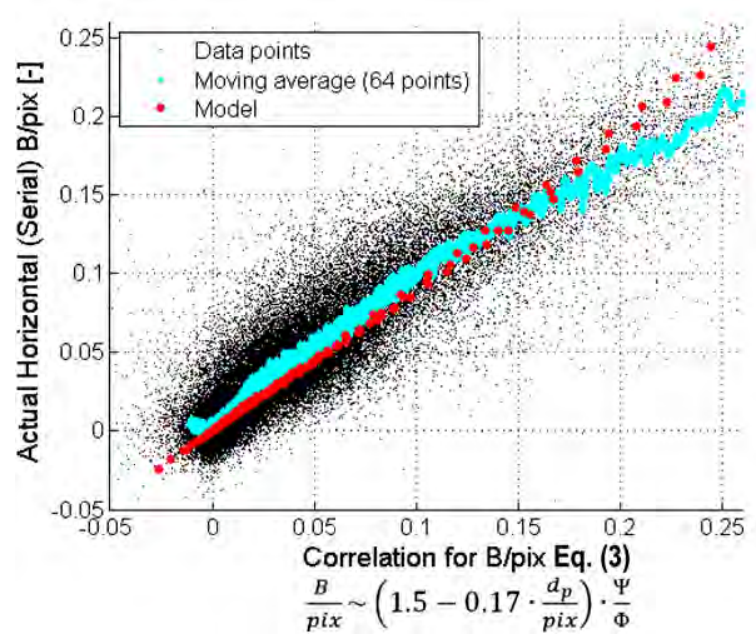

(a)

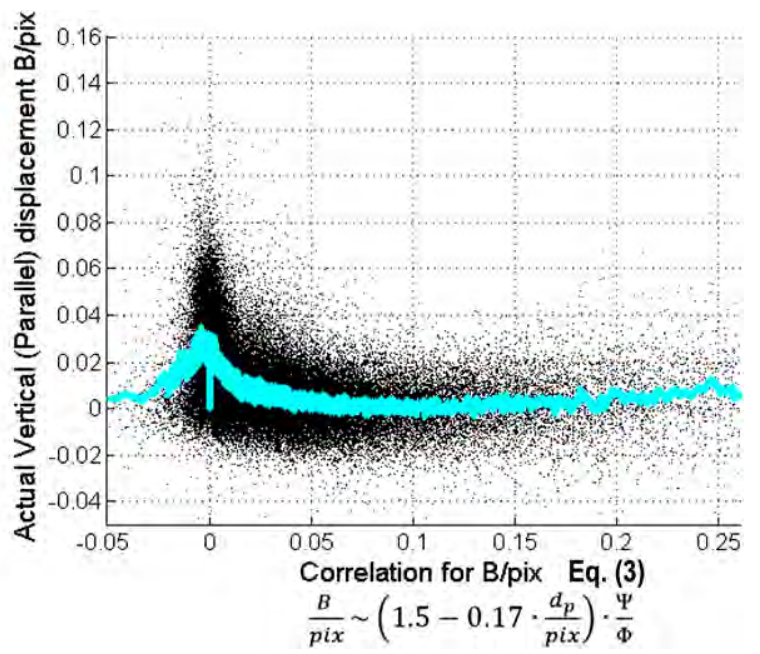

(b)

Figure 9. Measured displacement bias error $B$ for each PIV grid location in quadrant Q1. The rms value of the dispersion around the moving average is $\sim 0.015$ pixels for $(a)$ and $\sim 0.01$ for $(b)$.

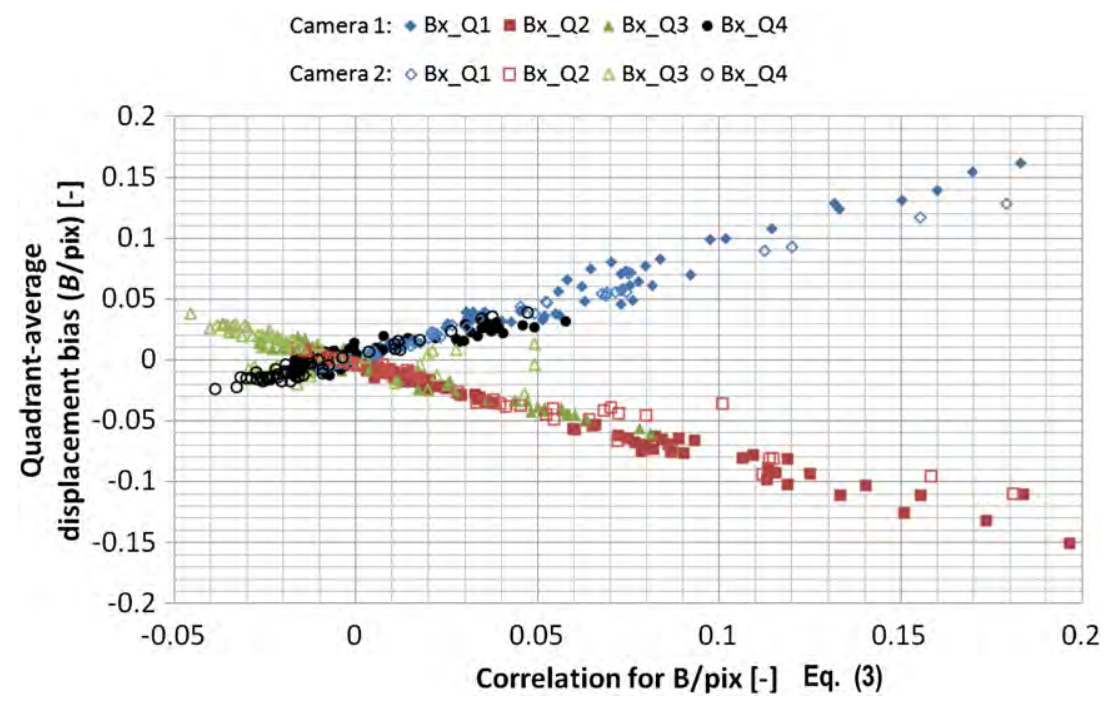

Figure 10. Average measured displacement bias error per quadrant for both MegaPlus cameras $\Delta I_{\max }$ and $I_{\max }$ both in gray levels for 12-bit cameras.

tested camera. Quadrants Q1 and Q4 exhibit similar positive displacement biases, which is coherent with the fact their serial shifts are in the same direction. In opposition, quadrants Q2 and Q3 show similar negative displacement biases, in the opposite direction to Q1 and Q4, in agreement with the general layout of the CCD sensor readout presented in figure 7 .

The magnitudes of the bias error are very similar for camera 1 and camera 2, as well as for left or right quadrants. Typical values of $\Delta I_{\max } \cdot I_{\max }^{-1.5} \sim 0.02$ yield horizontal displacement bias errors $B$ of the order of $\sim 0.05$ pixels, which are not negligible for PIV applications.

For different camera models the readout bias error may be different, but with similar architecture would follow the same principles, thus allowing for calibrating camera error. In particular, once the procedure in this section has validated equation (3) and it has been established that temperature and humidity effects are secondary, the values of the parameters $I_{\text {ref }}$ and $a$ can be easily assessed for a particular camera model. To do this, the $\Delta t$ can be reduced so that the real displacements are negligible; a double frame acquisition can provide the average value of $B$ for the camera (if it has a single readout structure) or for each quadrant (if it is a multi-quadrant one). For this acquisition, an average $\Delta I_{\max }$ and $I_{\max }$ can be obtained by checking the gray levels at the center of the particles (local maxima) in each frame. A few of this kind of acquisition changing laser power between first and second images can be implemented in equation (3) to provide the researcher with the values of $I_{\text {ref }}$ and $a$ for the cameras. This knowledge allows for an improved design of the experimental PIV acquisition procedure: i.e., if a multiple $\Delta t$ strategy is proposed for bias error correction in this particular application (Legrand et al 2011), the amount of error corresponding to the CCD readout can be established $a$ priori.

In addition, this camera calibration is relevant as far as it allows us to establish the values of $\Delta I_{\max }$ and $I_{\max }$ for which the readout error bias is acceptable in a particular application.

Increasing the detail of the assessment in respect to the average readout values given in figures 10,11 presents the 

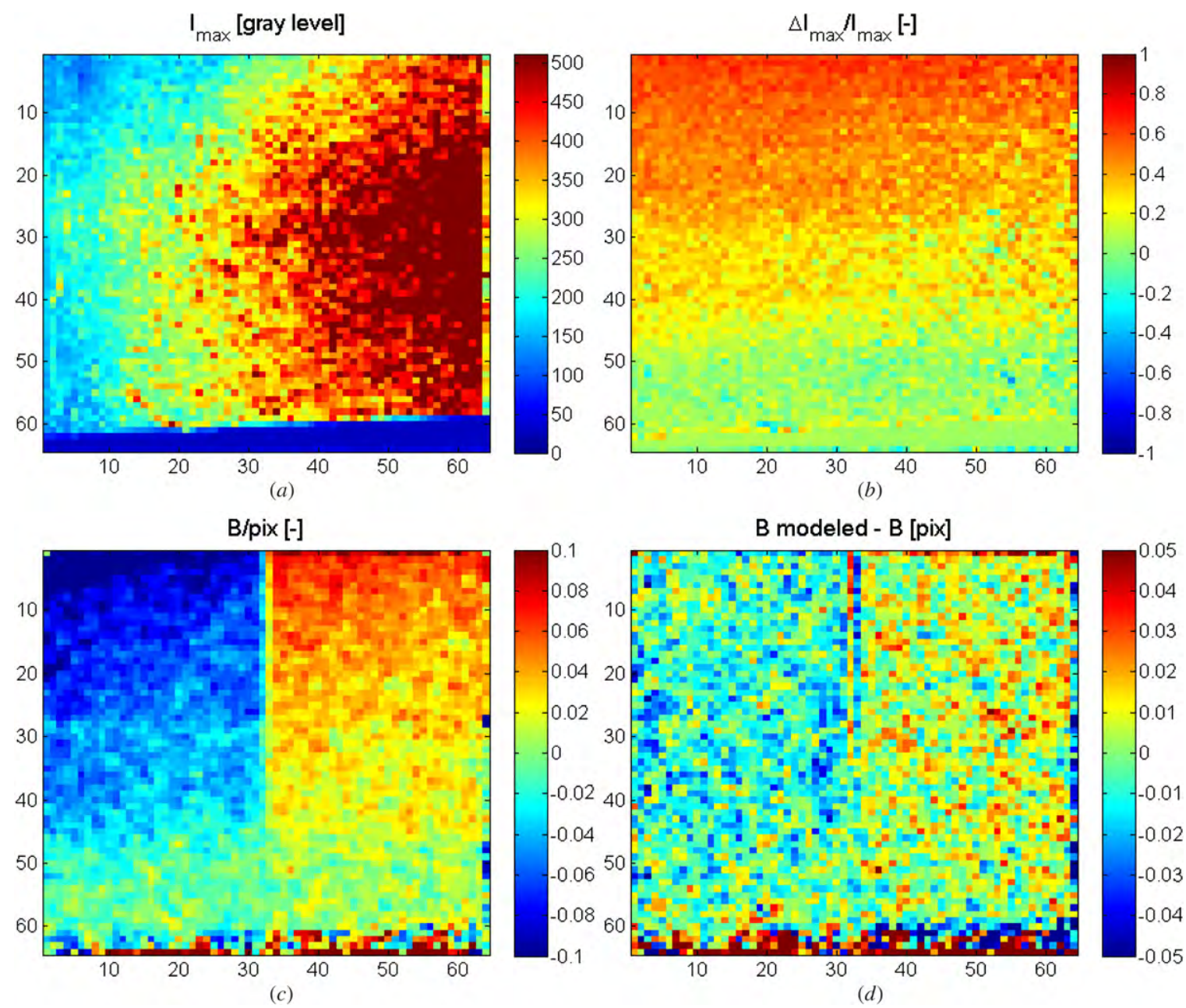

Figure 11. Local characteristics of a 200 images series. (a) Local $I_{\max }$ [gray levels]; (b) local $\Delta I_{\max } / I_{\max } \cdot[-] ;(c) B$ [pixels]; (d) local difference between the prediction from equation (3) and the real value of $B$ [pixels].

average measured characteristics at each PIV grid node along the whole view under test, for a series of 200 images $(T=$ $20{ }^{\circ} \mathrm{C}$, relative humidity $60 \%$, laser intensity $70 \%$ of maximum $400 \mathrm{~mJ})$. This figure depicts the spatial variation of the local $I_{\max }$ (figure 11(a)), the local $\Delta I_{\max } / I_{\max }$ (figure 11(b)), the local bias error $B$ (figure $11(c)$ ), and the local difference between the prediction from equation (3) and the real bias error, $B$, across the CCD chip for a certain PIV setup.

The $I_{\max }$ light intensity variations across the CCD chip in figure 11(a) corresponds to laser sheet light from right to left. Across the horizontal direction, the effect of the combination of the light extinction by the seeding particles and the divergence of the laser sheet is clearly appreciable. Along the vertical direction, the Gaussian shape intensity profiles of the laser sheets are noticeable. Although it is somewhat irregular, the difference between illumination pulses in figure $11(b)$ is coherent with two Gaussian profiles overlapping but with noncoincident axes, indicating a slight misalignment of the laser beam centers, magnified by the cylindrical lens that generates the laser sheets. Even though the two laser heads are tuned to emit the same amount of energy, the mentioned misalignment generates differences on the local relative illumination in the region of interest for the two different acquisition times separated by $\Delta t$.

Figure 11(c) shows that the horizontal displacement bias error may strongly vary across each quadrant, revealing a bias magnitude that can be locally much higher than the average given in figure 10. Left quadrants (Q2 and Q3) and right quadrants (Q1 and Q4) are easily distinguishable with their opposite horizontal bias error, as commented in the previous paragraphs. Finally, the difference between the predictions from equation (3) and the real bias error, $B$, plotted in figure $11(d)$, shows that this magnitude can be small enough to use the predictions for measurement corrections.

As left and right quadrants have opposite readout directions, particle displacements in the vicinity of the quadrant boundaries can be increased or mitigated depending on displacement direction (e.g. particle images recorded in Q1 in the first exposure and in a different quadrant in the second exposure). This effect is more apparent for large displacements between laser pulses and small interrogation windows. Nogueira et al (2009) have already discussed this artifact in their work and proposed a solution for minimizing errors in these regions through a multiple $\Delta t$ strategy in the measurement campaign. Besides, this affects a small portion 
of the whole chip area (typically less than $1 \%$ ), and it has been left out of the scope of the present work.

Results from figures 9-11 indicate that $\Delta I_{\max }$ can generate significant error from the readout procedure. It follows that laser illumination quality is of crucial importance for PIV applications. State-of-the-art lasers do not guarantee a perfect matching between the beam profiles from the two laser heads, and this makes it almost unavoidable to have local laser illumination differences in a PIV experiment. Even if this problem is overcome, it is impossible to generate perfect top-hat profiles for the laser sheet. In consequence, the movement of the particles within the laser sheet also generates illumination differences, as commented in section 3.1. In conclusion, readout displacement bias error should occur in virtually any PIV application.

In addition to this, the second CCD exposure is usually much longer than the first one, and may receive more stray background light than the first one. The associated illumination differences would generate bias errors in the readout procedure. The conclusion leads to highlighting the importance of avoiding spurious sources of light and the use of interferometric filters as means to reduce measurement errors.

Nogueira et al (2009) as well as Legrand et al (2011) have already discussed the feasibility of a multiple $\Delta t$ strategy designed to evaluate the bias error, including the possibility of correcting it under certain circumstances. However, the present work sheds light on the physical phenomenon of particle image smearing, and predicts the magnitude and whether or not some readout displacement bias error would be present in the measurements. This readout error can combine with other PIV errors (e.g. peak locking, Legrand et al (2011)), the resulting error being the same order of magnitude: $\sim 0.1$ pixel.

\section{Conclusions}

Previous studies on PIV applications have shown that stateof-the-art CCD cameras induce a bias error in the measured velocity. This work studies the architecture and the readout procedure of this kind of camera, widely used in PIV, with the aim of explaining the source of this bias. A predictive model has been elaborated considering that the charge transfer inefficiencies during the readout are especially relevant at the floating diffusion gate, which converts the accumulated charges into a voltage. This model is coherent with the bias errors observed in PIV measurements, related to smear of the particle images along the serial charge transfer direction. The expression obtained for the bias error, $B$, is

$$
\frac{B}{\text { pix }} \sim\left(1.5-0.17 \cdot \frac{d_{\mathrm{p}}}{\text { pix }}\right) \cdot \frac{\Psi}{\Phi} .
$$

This expression indicates that only two camera parameters $\left(I_{\text {ref }}\right.$ and $a$ ) are needed to evaluate the bias error produced by illumination differences between two PIV frames.

The expression has been validated for two 12-bit-depth interline CCD cameras (Kodak KAI-4000M CCD sensor, 4 megapixels provided with micro-lenses). Simple procedures to obtain the mentioned camera parameters are indicated, providing a characterization of these cameras with respect to this error, in which $I_{\text {ref }}=18.7 ; a=0.55$.

Tests indicate that the influence of the humidity and temperature of the working environment of the cameras on the error (smaller than $\sim 0.015$ pixels) is secondary in respect to the effect of the illumination differences. For common values of this parameter $\left(\Delta I_{\max } / I_{\max } \sim 0.2\right)$, the displacement bias error has been shown to be significant with this camera model (of the order of $\sim 0.05$ pixels).

As illumination differences between two laser pulses are almost unavoidable in a contemporary PIV application, the CCD readout displacement bias error will occur in almost any experiment. Results from different laboratories and different cameras, providing further validation and the values of $I_{\text {ref }}$ and $a$ for each model, would constitute relevant information, allowing us to optimize the design of the PIV acquisition procedure for a certain setup.

PIV cameras are expensive devices and a particular laboratory does not usually have many different models; this study focuses on the characterization of two cameras of the same model, but it indicates the procedure for other PIV researchers to perform the same kind of characterization for different PIV CCD cameras among the many types available. Results from different laboratories and different cameras would constitute relevant information in the future, allowing for a better design of the PIV acquisition procedure.

\section{Acknowledgments}

This work has been partially funded by the Spanish Research Agency grants ENE2006-13617 'TERMOPIV: Combustión y transferencia de calor analizadas con PIV avanzado' and ENE2011-28024 'TERMOPIV II', the regional grant CCG08UC3M/ENE-4432 'PIVEROT: Mejora en la medida PIV de campos fluidos de quemadores LSB por reducción de errores producidos por efectos termopsicrométricos en los sensores CCD' and the Madrid Community grant CCG10UC3M/ENE-5126 'ES-COMB: Estructuras coherentes en quemadores de combustión limpia' We would like also to express a special acknowledgement to the laboratory technicians Manuel Santos and Carlos Cobos, for their appreciated help in the experimental setup elaboration.

\section{References}

Burkey B C, Chang W C, Littlehale J, Lee T H, Tredwell T J, Lavine J P and Trabka E A 1984 The pinned photodiode for interline-transfer CCD image sensor IEDM: Int. Electron Devices Meeting pp 28-31

Cho Y C 1989 Digital image velocimetry Appl. Opt. 28 740-8

Grant I 1997 Particle image velocimetry: a review Proc. Instn. Mech. Eng. C 211 55-76

Grant I and Smith G H 1988 Modern developments in particle image velocimetry Opt. Lasers Eng. 9 245-64

Janesick J and Putnam G 2003 Developments and applications of high-performance CCD and CMOS imaging arrays Annu. Rev. Nucl. Part. Sci. 53 263-300

Kholmatov A, Akselli B, Nasibov A and Nasibov H 2010 Subpixel centroid position error analysis in particle tracking velocimetry induced by the CCD pixel binning Proc. SPIE 7723 77231R 
Kodak 1999 Megapixel interline CCD image sensor Kodak KAI-4000M Performance Specification Revision 1 frm220.free.fr/tcs-709/Kodak/KAI4000M.pdf

Kodak 2008 Kodak image sensors Revision 2.0 MTD/PS-0218 www.kodak.com/ek/uploadedFiles/chargeCoupledDevice.pdf

Kodak 2011 Image sensor device Kodak KAI-4011 Performance Specification KAI-4011LongSpec.pdf, revision 4.0 MTD/PS-0718 www.kodak.com/ek/US/en/KAI4011LongSpec.htm

Kompenhans J and Reichmuth J 1986 Particle imaging Velocimetry in a low turbulent wind tunnel and other flow facilities Proc. AGARD Conf. on Advanced Instrumentation for Aero Engine Components (Philadelphia, PA, USA 19-23 May) AGARD-CP 399-35

Lecuona A, Nogueira J, Rodríguez P A and Acosta A 2004 PIV evaluation algorithms for industrial applications Meas. Sci. Technol. 15 1027-38

Legrand M, Nogueira J, Lecuona A, Nauri S and Rodriguez P A 2010 Atmospheric low swirl burner flow characterization with stereo-PIV Exp. Fluids 48 901-13

Legrand M, Nogueira J, Ventas R and Lecuona A 2011 Simultaneous assessment of peak-locking and CCD readout errors through a multiple $\Delta t$ strategy Exp. Fluids 53 121-35

Lourenço L M, Krothapalli A, Buchlin J M and Riethmuller M L 1986 A non-invasive experimental technique for the measurement of unsteady velocity and vorticity fields AIAA $J$. 24 1715-7

Nobach H 2011 Influence of individual variations of particle image intensities on high-resolution PIV Exp. Fluids 50 919-27

Nobach H and Bodenschatz E 2009 Limitations of accuracy in PIV due to individual variations of particle image intensities Exp. Fluids 47 27-38
Nogueira J, Lecuona A, Nauri S, Legrand M and Rodríguez P A 2009 Multiple $\Delta t$ strategy for PIV error correction in a hot propulsive jet facility Meas. Sci. Technol. 20074001

Nogueira J, Lecuona A, Nauri S, Legrand M and Rodríguez P A 2011 Quantitative evaluation of PIV peak-locking through a multiple $\Delta t$ strategy: relevance of the rms component Exp. Fluids $\mathbf{5 1}$ 785-93

Nogueira J, Lecuona A and Rodríguez P A 2001 Identification of a new source of peak-locking, analysis and its removal in conventional and super-resolution PIV techniques Exp. Fluids 30 309-16

Raffel M, Willert C E and Kompenhans J 1998 Particle Image Velocimetry: A Practical Guide (Berlin: Springer)

Teranishi N and Ishihara Y 1987 Smear reduction in the interline CCD image sensor IEEE Trans. Electron Devices 34 1052-6

Theuwissen A 1995 Solid-State Imaging with Charge-Coupled Devices (Dordrecht: Kluwer)

Waczynski A, Polidan E J, Marshall P W, Reed R A, Johnson S D, Hill R J, Delo G S, Wassell E J and Cheng E S 2001 A comparison of charge transfer efficiency measurement techniques on proton damaged n-channel CCDs for the Hubble Space Telescope wide-field camera 3 IEEE Trans. Nucl. Sci. 48 1807-14

Wernet M 1991 Particle displacement tracking applied to air flows Laser Anemometry-Advances and Applications ed A Dybbs and B Ghorashi (Cleveland, OH: ASME) pp 327-36

Westerweel J 2000 Effect of sensor geometry on the performance of PIV interrogation Laser Techniques Applied to Fluid Mechanics ed R J Adrian et al (Berlin: Springer) pp 37-55

Whitmore B, Heyer I and Casertano S 1999 Charge-transfer efficiency of WFPC2 Publ. Astron. Soc. Pac. 111 1559-76

Willert C and Gharib M 1991 Digital particle image velocimetry Exp. Fluids 10 181-93 